\title{
Study on Consumer Exposure to Sun Spray and Sun Cream in South Korea
}

\author{
Mihyun Oh, Seoyoung Kim, Jieun Han, Sodam Park, Go Un Kim and Susun An \\ Safety \& Regulatory Research Division, AmorePacific Corporation R\&D Center, Yongin, Korea
}

\begin{abstract}
When conducting risk assessments of cosmetic ingredients, it is important that reliable exposure information is obtained for cosmetic products. As cosmetics are becoming more diverse, continuous effort must be made to obtain exposure data that reflect their growth and usage trends. The usage pattern of cosmetics, such as the application area and amount used, may differ by product type and also by country. We conducted a survey to compare the amount of sun spray and sun cream used in a usage environment in South Korea. The study was conducted on Haeundae Beach, one of the most popular beaches in South Korea. A total of 1,255 beachgoers participated in this study; 604 and 651 participants used the sun spray and sun cream, respectively, while sunbathing and enjoying water activities on the beach for one day. Exposure was analyzed following a probabilistic method. On comparing all subjects, it was found that the group that used sun spray (mean: $44.52 \mathrm{~g} /$ day) used significantly more product $(p=0.000)$ than those who used sun cream (mean: $20.51 \mathrm{~g} /$ day). By analyzing the daily exposure of sun spray and sun cream per unit body weight according to age and gender, the exposure amount of sun spray and sun cream was found to be highest among 2 9 year-old girls (mean for sun spray: $2.51 \mathrm{~g} / \mathrm{kg} / \mathrm{day}, \mathrm{p} 95: 5.50 \mathrm{~g} / \mathrm{kg} / \mathrm{day}$, mean for sun cream: $0.79 \mathrm{~g} / \mathrm{kg} /$ day, p95: $1.79 \mathrm{~g} / \mathrm{kg} /$ day). The amount of sun spray used is approximately twice that of sun cream. Among both the sun spray and sun cream groups, the exposure amount per unit body weight was highest in girls younger than 10. These factors should be considered when conducting risk assessments of sun spray and sun cream.
\end{abstract}

Key words: Sun spray, Sun cream, Consumer Exposure amount, South Korea, Beach

\section{INTRODUCTION}

Safety evaluation for cosmetic products and their components is essential because such products are used in diverse environments, and they are applied directly onto the skin. During the risk assessment of such cosmetic components, it is important to obtain reliable exposure information about the cosmetic products. Because cosmetics are becoming more diverse and people are using them in different ways, a continuous effort must be made to

Correspondence to: Susun An, Safety \& Regulatory Research Division, AmorePacific Corporation R\&D Center, 1920, Yonggudaero, Giheung-gu, Yongin-si, Gyeonggi-do 17074, Korea

E-mail: ssan@amorepacific.com

This is an Open-Access article distributed under the terms of the Creative Commons Attribution Non-Commercial License (http:// creativecommons.org/licenses/by-nc/3.0) which permits unrestricted non-commercial use, distribution, and reproduction in any medium, provided the original work is properly cited. obtain exposure data reflecting such trends.

Europe and the U.S. have been conducting consumer exposure research on various cosmetic categories for several years. In Europe, an exposure evaluation of body lotion, deodorant/antiperspirant in aerosol and non-aerosol forms, lipstick, facial moisturizer, shampoo, and toothpaste was conducted in 2003 and 2005 (1,2). Furthermore, in 2007-2008, research was conducted on hair styling, hand cream, liquid foundation, mouthwash, and shower gel (3). The U.S. Cosmetic, Toiletry, and Fragrance Association conducted an exposure evaluation of hairspray, spray perfume, liquid foundation, shampoo, and eye shadow $(4,5)$.

Sunscreen is a cosmetic product that is used consistently because of the generally high level of interest in sun blocking, and research has previously been conducted on its usage amount. According to previous literature, under realistic conditions on the beach using sun products applied to the entire body, product usage values of $0.5-1.3 \mathrm{mg} / \mathrm{cm}$ have been reported (6-11). Recently, there has been an 
increase in various types of products for easier application to the entire body surface or topical areas with the development of products such as sun lotions, sun creams, sprays, sticks, balms, and cushions. The ease of application leads to high levels of sun spray usage, especially during the summer season, and frequent use of sun spray during outdoor activities.

Even among products with the common purpose of sun blocking, usage pattern parameters such as application area, frequency, and amount of usage may differ by product category. Usage patterns may also vary by country. Despite this, there have been few studies on the amount of exposure of current sun sprays in actual consumer environments, especially in Asia. To fill this gap, we conducted a comparison study on sun spray and sun cream usage in an actual consumer environment in South Korea.

\section{MATERIALS AND METHODS}

Study design. The study was conducted at Haeundae Beach, which is one of the most visited and popular beaches in South Korea. In August 2015, we recruited beachgoing volunteers aged between 2 and 60 years old who enjoyed sunbathing and water activities. According to the Korea Meteorological Administration, the average temperature during the study period was $27-29^{\circ} \mathrm{C}$, and the relative humidity was $75-80 \%$.

On the day of survey, people who visited the beach in the morning and stayed there for more than half the day were recruited. Subjects who had pre-existing skin diseases (e.g., eczema, psoriasis) or very sensitive skin, especially to sunscreen, were excluded from the study. Children were enrolled with parental permission. Sun cream and sun spray with the same sun protection factor (SPF50+, $\mathrm{PA}+++$ ) were selected for this study (active ingredients in sun cream: ethylhexyl methoxycinnamate (CAS No. 546677-3), diethylamino hydroxybenzoyl hexyl benzoate (CAS No. 302776-68-7), titanium dioxide (CAS No. 13463-677), isoamyl p-methoxycinnamate (CAS No. 71617-10-2); active ingredients in sun spray: ethylhexyl methoxycinnamate (CAS No. 5466-77-3), zinc oxide (CAS No. 131413-2), ethylhexyl salicylate (CAS No. 118-60-5), isoamyl p-methoxycinnamate (CAS No. 71617-10-2), bis-ethylhexyloxyphenol methoxyphenyl triazine (CAS No. 18739300-6)). The subjects randomly received a sun cream or a sun spray. For subjects under the age of 10, we provided products for children and asked the parents to apply the product to the child's body. The product weight was measured before use and participants were asked to fill out a log specifying the time of the day and the body parts to which the product was applied. When the subjects were leaving the beach, the sunscreens were weighed again, and the logs were collected. The study protocol was approved by the AmorePacific institutional review board.
Questionnaire. Enrolled subjects were asked to fill out personal information, including age and gender, for statistical analysis. They were also asked to provide body weight to enable us to calculate the amount of sunscreen used per unit body weight. In addition, participants filled out a usage pattern questionnaire.

Statistical analyses. We followed the Monte Carlo simulation method to allow a larger sample size for estimating more realistic exposure across a population. The bestfitting distribution was determined by fitting parametric distributions (normal, lognormal, beta, gamma, etc.) to the measured experimental data. The goodness-of-fit for each distribution was assessed using the Anderson-Darling (AD) goodness-of-fit test, $\chi 2$-test, and Kolmogorov-Smirnov test using Oracle C Crystal Ball software. The product usage amount was obtained by multiplying the distribution by 10,000 iterations using Monte Carlo random simulations (Crystal Ball software, Oracle Corp., CA, USA).

The independent t-test was used to assess the differences between the two groups (sun spray and sun cream, male and female) and was derived using SPSS $₫$ for Windows computer software (SPSS Inc., IL, USA). Statistical significance was defined as $p<0.05$.

\section{RESULTS}

A total of 1,255 subjects participated in the study; 604

Table 1. Age distribution of the study population $(n=1,255)$

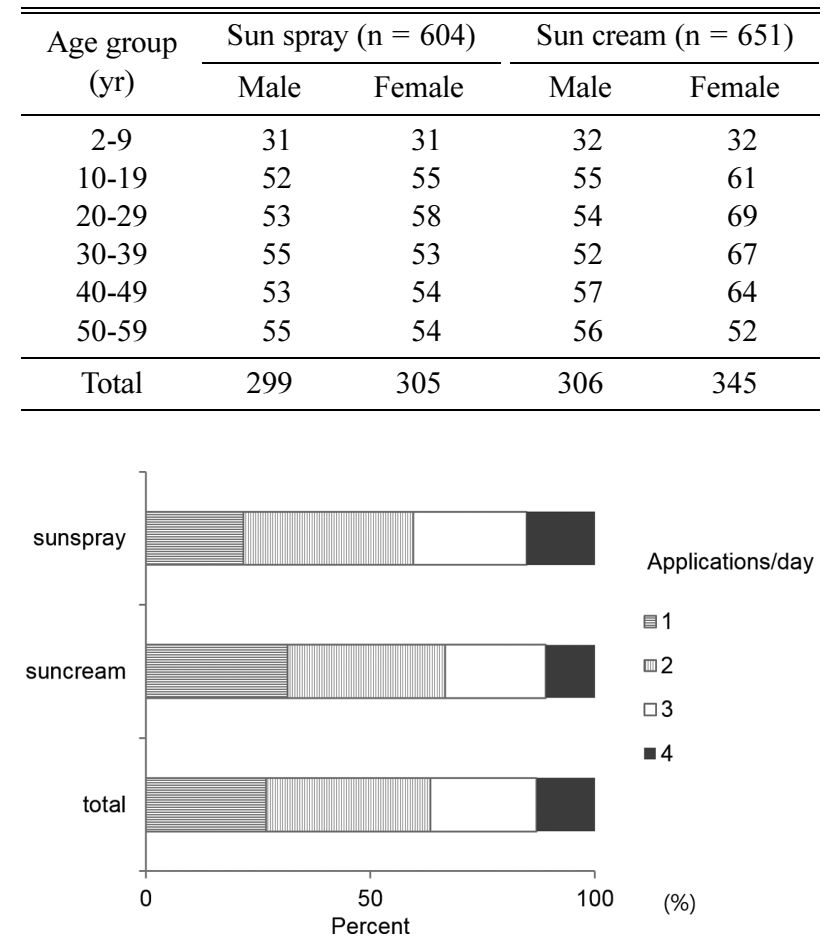

Fig. 1. Number of applications of sunscreen per study day. 
Table 2. Average number of sunscreen applications per study day

\begin{tabular}{lcc}
\hline \hline Statistics & Sun spray & Sun cream \\
\hline $\mathrm{N}$ & 604 & 651 \\
Mean & 2.34 & 2.13 \\
Std. deviation & 0.040 & 0.038 \\
$p$-Value & & 0.000 \\
\hline
\end{tabular}

participants used the sun spray and 651 participants used the sun cream. Detailed information on the subjects by age and gender is presented in Table 1 .

Frequency of use. The distribution of the use frequency for sun spray and sun cream is provided in Fig. 1. The mean use frequency for sun spray and sun cream were 2.34 and 2.13, respectively (Table 2). Subjects who used the sun spray showed a higher frequency of use than those who used the sun cream $(p<0.01)$. A gender comparison revealed that male and female subjects showed no significant difference in the frequency of use (data not shown).

Application area. While using either the sun cream or sun spray, participants were asked to maintain a log of the body parts the product was applied to. The survey analysis showed that sun cream was applied most frequently on the face $(68.5 \%)$, followed by the arms $(64.5 \%)$, legs (49.5\%), and trunk (24.3\%) (Fig. 2). On the other hand, sun spray was most commonly applied on the legs and arms (63.9\% and $62.5 \%$, respectively), followed by the face and trunk (37.6\% and $17.7 \%$, respectively). The largest difference was in the face category, where sun cream was applied the most $(68.5 \%)$, but sun spray was applied less $(37.6 \%)$.

Amount of use. A comparison of all subjects revealed that the sun spray group (mean score: $44.52 \mathrm{~g} /$ day) used the product significantly more $(p=0.000)$ than the sun

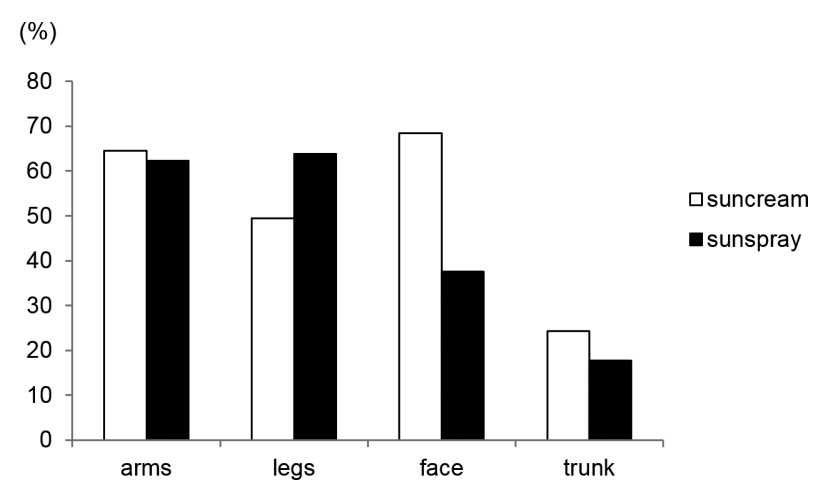

Fig. 2. Body parts to which sunscreen was applied on the study day.
Table 3. Amount of sun spray and sun cream applied per unit body weight (g/day, g/kg/day)

\begin{tabular}{lccrrr}
\hline \hline & \multicolumn{2}{c}{ Sun spray } & & \multicolumn{2}{c}{ Sun cream } \\
\cline { 2 - 3 } \cline { 5 - 6 } & g/day & g/kg/day & & g/day & g/kg/day \\
\hline $\mathrm{N}$ & \multicolumn{2}{c}{604} & & \multicolumn{2}{c}{651} \\
Mean & 44.52 & 0.77 & & 20.51 & 0.38 \\
Std. deviation & 23.83 & 0.65 & & 8.85 & 0.22 \\
Percentile & & & & \\
10th & 20.13 & 0.34 & & 12.09 & 0.19 \\
20th & 26.67 & 0.45 & & 13.43 & 0.22 \\
30th & 32.41 & 0.55 & & 14.76 & 0.25 \\
40th & 38.44 & 0.65 & & 16.26 & 0.29 \\
50th & 44.91 & 0.77 & & 17.95 & 0.32 \\
60th & 51.78 & 0.90 & & 20.07 & 0.37 \\
70th & 59.85 & 1.07 & & 22.82 & 0.42 \\
80th & 69.46 & 1.31 & & 26.41 & 0.50 \\
90th & 82.95 & 1.70 & 32.28 & 0.63 \\
95th & 93.82 & 2.13 & 38.21 & 0.80 \\
\hline
\end{tabular}

cream group (mean score: $20.51 \mathrm{~g} /$ day), which was more than twice the mean score (Table 3).

Analyzing the amount of sun spray and sun cream usage according to age, the group with the highest mean usage score was the 10-19 year age group (sun spray men: 51.93 g/day; sun cream men: $23.10 \mathrm{~g}$ /day; sun cream women: $23.06 \mathrm{~g}$ /day), followed by the 20-59 year age group (sun spray men: $47.29 \mathrm{~g} /$ day; sun cream men: $21.07 \mathrm{~g} /$ day; sun cream women: $20.31 \mathrm{~g} /$ day), and the 2-9 year age group (sun spray men: $40.25 \mathrm{~g}$ /day; sun cream men: $18.53 \mathrm{~g}$ /day; sun cream women: $16.48 \mathrm{~g} /$ day). However, the sun spray women groups showed different usage patterns, with the amount of usage being similar in all age groups (mean score: 48.59-48.85 g/day) (Table 4-7).

We also analyzed the amount of sunscreen applied per

Table 4. Amount of sun spray applied per use day by gender and age (g/day)

\begin{tabular}{llllllll}
\hline \hline & \multicolumn{3}{c}{ Male (yr) } & & \multicolumn{3}{c}{ Female (yr) } \\
\cline { 2 - 5 } \cline { 7 - 8 } & $2-9$ & $10-19$ & $20-59$ & & $2-9$ & $10-19$ & $20-59$ \\
\hline $\mathrm{N}$ & 31 & 52 & 216 & & 31 & 55 & 219 \\
Mean & 40.25 & 51.93 & 47.29 & & 48.85 & 48.85 & 48.59 \\
Std. deviation & 22.50 & 33.82 & 24.21 & & 22.56 & 22.56 & 22.50 \\
$\begin{array}{l}\text { Percentile } \\
\text { 10th }\end{array}$ & 20.44 & 17.52 & 19.63 & & 17.55 & 29.61 & 21.27 \\
20th & 23.71 & 25.05 & 26.17 & & 25.28 & 36.05 & 28.06 \\
30th & 27.02 & 31.76 & 31.65 & & 33.16 & 42.60 & 33.85 \\
40th & 30.48 & 38.05 & 37.15 & & 41.12 & 49.36 & 39.82 \\
50th & 34.24 & 44.79 & 42.83 & & 48.90 & 56.13 & 45.80 \\
60th & 38.49 & 52.47 & 49.33 & & 56.97 & 63.47 & 51.98 \\
70th & 44.24 & 61.82 & 56.61 & & 64.72 & 71.93 & 59.06 \\
80th & 52.53 & 73.75 & 66.37 & & 72.48 & 81.69 & 67.57 \\
90th & 66.78 & 94.26 & 80.64 & & 79.95 & 94.98 & 80.80 \\
95th & 82.98 & 115.35 & 92.73 & & 83.67 & 104.79 & 90.42 \\
\hline
\end{tabular}


Table 5. Exposure to sun spray per use day by gender and age $(\mathrm{g} / \mathrm{kg} /$ day $)$

\begin{tabular}{lcccccc}
\hline \hline & \multicolumn{3}{c}{ Male (yr) } & \multicolumn{3}{c}{ Female (yr) } \\
\cline { 2 - 7 } & $2-9$ & $10-19$ & $20-59$ & $2-9$ & $10-19$ & $20-59$ \\
\hline $\mathrm{N}$ & 31 & 52 & 216 & 31 & 55 & 219 \\
Mean & 1.39 & 0.83 & 0.66 & 2.51 & 1.24 & 0.88 \\
Std. deviation & 0.89 & 0.49 & 0.33 & 1.53 & 0.68 & 0.43 \\
Percentile & & & & & & \\
10th & 0.49 & 0.23 & 0.29 & 0.81 & 0.61 & 0.38 \\
20th & 0.69 & 0.35 & 0.37 & 1.13 & 0.73 & 0.50 \\
30th & 0.85 & 0.46 & 0.45 & 1.45 & 0.84 & 0.60 \\
40th & 1.01 & 0.60 & 0.53 & 1.79 & 0.96 & 0.70 \\
50th & 1.18 & 0.74 & 0.61 & 2.17 & 1.07 & 0.81 \\
60th & 1.39 & 0.90 & 0.69 & 2.62 & 1.22 & 0.92 \\
70th & 1.62 & 1.08 & 0.80 & 3.12 & 1.39 & 1.05 \\
80th & 1.96 & 1.30 & 0.92 & 3.78 & 1.63 & 1.21 \\
90th & 2.51 & 1.56 & 1.12 & 4.74 & 2.07 & 1.46 \\
95th & 3.05 & 1.75 & 1.29 & 5.50 & 2.52 & 1.68 \\
\hline
\end{tabular}

Table 6. Amount of sun cream applied per use day by gender and age (g/day)

\begin{tabular}{lrlllll}
\hline \hline & \multicolumn{3}{c}{ Male (yr) } & \multicolumn{3}{c}{ Female (yr) } \\
\cline { 2 - 7 } & \multicolumn{1}{c}{$2-9$} & $10-19$ & $20-59$ & \multicolumn{1}{c}{$2-9$} & $10-19$ & $20-59$ \\
\hline $\mathrm{N}$ & 32 & 55 & 219 & 32 & 61 & \multicolumn{1}{c}{252} \\
Mean & 18.53 & 23.10 & 21.07 & 16.48 & 23.06 & 20.31 \\
Std. deviation & 5.31 & 14.89 & 11.17 & 4.75 & 17.73 & 8.08 \\
Percentile & & & & & & \\
10th & 12.17 & 12.93 & 12.55 & 12.31 & 12.18 & 12.08 \\
20th & 13.54 & 14.26 & 13.75 & 13.06 & 13.27 & 13.54 \\
30th & 14.83 & 15.57 & 14.93 & 13.72 & 14.51 & 15.02 \\
40th & 16.19 & 17.05 & 16.18 & 14.40 & 15.91 & 16.57 \\
50th & 17.61 & 18.77 & 17.74 & 15.19 & 17.68 & 18.37 \\
60th & 19.28 & 20.92 & 19.59 & 16.14 & 19.89 & 20.40 \\
70th & 21.15 & 23.87 & 22.09 & 17.27 & 23.17 & 22.75 \\
80th & 23.33 & 28.42 & 25.92 & 19.05 & 28.00 & 25.78 \\
90th & 26.27 & 37.49 & 33.40 & 22.16 & 38.07 & 31.24 \\
95th & 28.49 & 47.98 & 41.41 & 25.31 & 52.12 & 36.05 \\
\hline
\end{tabular}

body weight, dividing the amount of product usage by the weight of the corresponding subjects. Comparing all subjects, the sun spray group (mean score: $0.77 \mathrm{~g} / \mathrm{kg} / \mathrm{day}$ ) used the product significantly more $(p=0.000)$ than the sun cream group (mean score: $0.38 \mathrm{~g} / \mathrm{kg} /$ day) (Table 3 ).

According to gender, the mean score of the amount of sun spray and sun cream usage per body weight showed that the 2-9 year age group applied the highest amount per unit body weight followed by the 10-19 and the 20-59 year age groups (Table 5, 7). Comparing the amount of usage per unit body weight according to gender, there was not a significant difference in all age groups for sun cream $(p>0.05)$. On the other hand, the amount of sun spray usage was significantly higher for women than men for all age groups $(p<0.01)$.
Table 7. Exposure to sun cream per use day by gender and age $(\mathrm{g} / \mathrm{kg} /$ day $)$

\begin{tabular}{lcccccc}
\hline \hline & \multicolumn{3}{c}{ Male (yr) } & \multicolumn{3}{c}{ Female (yr) } \\
\cline { 2 - 7 } & $2-9$ & $10-19$ & $20-59$ & $2-9$ & $10-19$ & $20-59$ \\
\hline $\mathrm{N}$ & 32 & 55 & 219 & 32 & 61 & 252 \\
Mean & 0.61 & 0.35 & 0.30 & 0.79 & 0.47 & 0.37 \\
Std. deviation & 0.29 & 0.22 & 0.14 & 0.55 & 0.33 & 0.15 \\
Percentile & & & & & & \\
10th & 0.30 & 0.19 & 0.17 & 0.37 & 0.23 & 0.21 \\
20th & 0.38 & 0.21 & 0.19 & 0.43 & 0.26 & 0.24 \\
30th & 0.45 & 0.23 & 0.21 & 0.49 & 0.29 & 0.28 \\
40th & 0.50 & 0.26 & 0.24 & 0.55 & 0.32 & 0.31 \\
50th & 0.56 & 0.29 & 0.26 & 0.63 & 0.37 & 0.34 \\
60th & 0.63 & 0.32 & 0.29 & 0.72 & 0.42 & 0.38 \\
70th & 0.71 & 0.37 & 0.32 & 0.84 & 0.49 & 0.42 \\
80th & 0.82 & 0.44 & 0.37 & 1.03 & 0.60 & 0.48 \\
90th & 0.99 & 0.57 & 0.47 & 1.37 & 0.81 & 0.57 \\
95th & 1.17 & 0.74 & 0.57 & 1.79 & 1.06 & 0.66 \\
\hline
\end{tabular}

\section{DISCUSSION}

We conducted a comparative study on the amount of sun spray and sun cream used by consumers in South Korea.

The frequency of use of sun spray (2.34 applications per day) was higher than that of sun cream (2.13 applications per day). One reason could be that the former is easier to re-apply compared to the latter; the differences in the frequency of use may have influenced the difference in the usage amount between the two types of sunscreen. Analyzing the area of cosmetic application, $37.6 \%$ of the sun spray was applied to the face, which was lower than the amount of sun cream applied to the face (68.5\%). However, the fact that approximately one-third of the subjects used sun spray on their faces means that significant attention should be directed toward the inhalation toxicity of sun spray products. Over the years, the cosmetics industry has been developing prediction models to estimate inhalation exposure using computer simulation programs and actual measured data $(12,13)$. It has been conducting research on particle size and behavior, as well as on the toxicity of spray ingredients to evaluate the risk of inhalation. It is also important to estimate the degree of exposure precisely according to user environment.

We verified the amount of daily sunscreen usage. A total of $20.51 \mathrm{~g} /$ day of sun cream was used, which is higher than the values reported in previous research conducted under similar conditions (beach) in Europe. As the exposure degrees of cosmetics vary among countries, research is imperative for each country to enable accurate risk assessment. The use of sun spray (44.52 g/day) was twice that of sun cream $(20.51 \mathrm{~g} /$ day $)$, and it is assumed that a larger amount of sun spray was applied to the entire body as it is easier to apply, and the amount applied is perceived as 
Table 8. Comparison of sunscreen use between previous studies and current study

\begin{tabular}{lccccc}
\hline \hline & Bech-Thomsen et al. (9) & Autier et al. (7) & SCCS (17) & \multicolumn{2}{c}{ Current study } \\
\hline Product & Sunscreen & Sunscreen & Sunscreen & Sun spray & Sun cream \\
Place & Beach & Resort & - & Beach \\
Amount used $\left(\mathrm{mg} \mathrm{cm}^{-2}\right)$ & 0.5 (mean) & 0.39 (median) & - & - & - \\
Amount used (g/day) & 17.5 (mean, calculated) & 13.7 (median, calculated) & 18.0 & 44.52 (mean) & 20.51 (mean) \\
\hline
\end{tabular}

lower than that of sun cream (Table 8).

An exposure assessment for different sunscreen categories, namely sun spray, sun cream, and sun oil, was conducted in the city of Brest, France, in 2016. In this study, the usage amount of sun spray was similar or less than that of sun cream, which differs from our findings (14). Specifically, the study conducted in France used a pump-type sun spray, and our research used propellant-type sun spray. Pump-type sun spray is released for a short period of time with one operation, while propellant-type sun spray is released continuously during use. Owing to this difference, propellant-type sun sprays release a larger amount of product. An exposure evaluation study of cosmetics was conducted in South Korea in 2016. In that study, dose of cosmetics exposure in babies up to the age of 36 months was assessed in two cities in South Korea during both winter and summer. Daily exposure per unit body weight obtained in that study was higher for males than for females. In our study, we obtained the opposite result, the sunscreen exposure for females was higher than that for males (15). Our study was conducted during one day of outdoor activity, and the study from 2016 was evaluated on a daily basis over a 14-day period, and these differences may have affected these results.

Estimating the degree of exposure by age and gender is an important aspect of risk assessment. We analyzed the sunscreen amount by age and gender. The use of sun spray per unit body weight according to age is highest among the 2-9-year age group, followed successively by the 1019 and the 20-59 year age groups. The usage per unit body weight in women is remarkably higher than that in men in all age groups $(p<0.01)$. As a result, sun spray exposure amount per unit body weight among all groups was highest in 2-9-year-old girls (mean score: $2.51 \mathrm{~g} / \mathrm{kg}$ /day, p95: $5.50 \mathrm{~g} / \mathrm{kg} /$ day). The recent research mentioned above states that sunscreen per $\mathrm{cm}^{2}$ applied by parents on their babies was higher than the amount used for adults (15). Another study on aggregate consumer exposure to the UV filter ethylhexyl methoxycinnamate revealed that children were subjected to the highest exposure (16). There was no mention of amount of usage for sunscreen in that study, but its results are similar to ours in that children are more exposed to sunscreen than adults, given that the ethylhexyl methoxycinnamate is the most common UV filter.

Nowadays, brands of cosmetics bring out various new products in line with the people's increased consumption patterns and improved technology. Sun spray is a type of sunscreen that is frequently used, not only in the U.S. and Europe, but also in Asia. The results of this study show that there is a gap in frequency of use and exposure amount in both sun spray and sun cream among consumers in South Korea. Therefore, it is necessary to strengthen risk assessment through exposure evaluations suitable for various product categories and user environments.

\section{CONFLICT OF INTEREST}

The authors have no conflict of interest to disclose.

Received December 3, 2018; Revised April 11, 2019; Accepted April 25, 2019

\section{REFERENCES}

1. Hall, B., Tozer, S., Safford, B., Coroama, M., Steiling, W., Leneveu-Duchemin, M.C., McNamara, C. and Gibney, M. (2007) European consumer exposure to cosmetic products, a framework for conducting population exposure assessments. Food Chem. Toxicol., 45, 2097-2108.

2. McNamara, C., Rohan, D., Golden, D., Gibney, M., Hall, B., Tozer, S., Safford, B., Coroama, M., Leneveu-Duchemin, M.C. and Steiling, W. (2007) Probabilistic modelling of European consumer exposure to cosmetic products. Food Chem. Toxicol., 45, 2086-2096.

3. Hall, B., Steiling, W., Safford, B., Coroama, M., Tozer, S., Firmani, C., McNamara, C. and Gibney, M. (2011) European consumer exposure to cosmetic products, a framework for conducting population exposure assessments Part 2. Food Chem. Toxicol., 49, 408-422.

4. Loretz, L., Api, A.M., Barraj, L., Burdick, J., Davis de, A., Dressler, W., Gilberti, E., Jarrett, G., Mann, S., Laurie Pan, Y.H., Re, T., Renskers, K., Scrafford, C. and Vater, S. (2006) Exposure data for personal care products: hairspray, spray perfume, liquid foundation, shampoo, body wash, and solid antiperspirant. Food Chem. Toxicol., 44, 2008-2018.

5. Loretz, L.J., Api, A.M., Babcock, L., Barraj, L.M., Burdick, J., Cater, K.C., Jarrett, G., Mann, S., Pan, Y.H., Re, T.A., Renskers, K.J. and Scrafford, C.G. (2008) Exposure data for cosmetic products: facial cleanser, hair conditioner, and eye shadow. Food Chem. Toxicol., 46, 1516-1524.

6. Gottlieb, A., Bourget, T.D. and Lowe, N.J. (1997) Sunscreens: effects of amounts of application of sun protection factors. Cosmet. Sci. Technol. Ser., 15, 583-588.

7. Autier, P., Boniol, M., Severi, G. and Doré, J.F.; European Organization for Research and Treatment of Cancer Mela- 
noma Co-operative Group (2001) Quantity of sunscreen used by European students. Br. J. Dermatol., 144, 288-291.

8. Stenberg, C. and Larko, O. (1985) Sunscreen application and its importance for the sun protection factor. Arch. Dermatol., 121, 1400-1402.

9. Bech-Thomsen, N. and Wulf, H.C. (1992) Sunbathers' application of sunscreen is probably inadequate to obtain the sun protection factor assigned to the preparation. Photodermatol. Photoimmunol. Photomed., 9, 242-244.

10. Autier, P., Boniol, M. and Doré, J.F. (2007) Sunscreen use and increased duration of intentional sun exposure: still a burning issue. Int. J. Cancer, 121, 1-5.

11. Diffey, B. (1996) Sunscreens, suntans and skin cancer. People do not apply enough sunscreen for protection. $B M J, \mathbf{3 1 3}$, 942.

12. Rothe, H., Fautz, R., Gerber, E., Neumann, L., Rettinger, K., Schuh, W. and Gronewold, C. (2011) Special aspects of cosmetic spray safety evaluations: principles on inhalation risk assessment. Toxicol. Lett., 205, 97-104.

13. Steiling, W., Bascompta, M., Carthew, P., Catalano, G., Corea, N., D’Haese, A., Jackson, P., Kromidas, L., Meurice,
P., Rothe, H. and Singal, M. (2014) Principle considerations for the risk assessment of sprayed consumer products. Toxicol. Lett., 227, 41-49.

14. Ficheux, A.S., Chevillotte, G., Wesolek, N., Morisset, T., Dornic, N., Bernard, A., Bertho, A., Romanet, A., Leroy, L., Mercat, A.C., Creusot, T., Simon, E. and Roudot, A.C. (2016) Consumption of cosmetic products by the French population second part: amount data. Food Chem. Toxicol., 90, 130-141.

15. Lee, E., Yun, J., Ha, J., Park, B.C., Park, G.H., Kim, H.R., Hong, S.P., Kim, K.B. and Kim, M.H. (2017) Assessment of exposure for baby cosmetic care products in a Korean population. Food Chem. Toxicol., 106, 107-113.

16. Manová, E., von Goetz, N. and Hungerbuehler, K. (2015) Aggregate consumer exposure to UV filter ethylhexyl methoxycinnamate via personal care products. Environ. Int., 74, 249257.

17. SCCS (2015) The SCCS Notes of Guidance for the Testing of Cosmetic Ingredients and their Safety Evaluation (9th revision). 